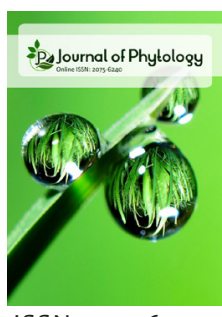

ISSN: $2075-6240$

\title{
Oral cancer preventive potential of Polydatin: A nanoencapsulation approach
}

\author{
Vijayalakshmi Sankaran', Santhosh Kumar Murali', Alaa Y Ghidan², \\ Tawfiq M Al-Antary³, Ernest David ${ }^{1 *}$
}

'Department of Biotechnology, Thiruvalluvar University, Serkadu, Vellore-632 115, Tamilnadu, India, ${ }^{2}$ Nanotechnology and Microbiology, Research and Development Center, The Higher Council for Science and Technology, Amman 11941, Jordan, 3'Department of Plant protection, Faculty of Agriculture, University of Jordan, Amman, 11942, Jordan

Received: August 24, 2020

Revised: December 04, 2020

Accepted: December 11, 2020

Published: December 23, 2020

*Corresponding Author:

Ernest David

E-mail: ernestdavid2009@gmail.

com

\section{ABSTRACT}

Over the period, several medicinal plants are recognized as attractive sources of anti-cancer molecules. About 35,000 plant species have been tested for possible anticancer activities by the National Cancer Center (NCI). Among them, reproducible anticancer behavior has been shown by around 3,000 plant species. Based on this information, we aimed to investigate the cancer preventive efficiency of Polydatin encapsulated PLGA nanoparticles (POL-PLGA-NPs) against 7,12 dimethylbenz (a) anthracene (DMBA) induced buccal pouch carcinogenesis by modulating the antioxidants process in male Syrian hamsters. Oral cancer was initiated on left side buccal pouches by applying DMBA (0.5\%), (a known potent chemical carcinogen) on alternative days in a week (thrice a week) for a period of 14 weeks to provoke oral cancer. Sequences of oral squamous cell carcinoma are noted to be manifested exhibiting the formation of incidence, size, mass and development of tumor presented as preneoplastic and neoplastic lesions in hamsters oral buccal pouch tissues. At the same time, oral administration of POL-PLGA-NPs $(30 \mathrm{mg} / \mathrm{kg}$.b.wt.) considerably increased glutathione peroxidase (GPx) and glutathione (GSH) levels in the buccal tissue thereby decreasing lipid peroxidation activity which resulted in neoplasms incidence in buccal tissue of DMBA painted animals. Thus, POL-PLGA-NPs activity seemed to be a potent cancer preventive agent.

KEYWORDS: Medicinal plant, Polyphenol, Polydatin, Hamster

\section{INTRODUCTION}

Oral cancer is a general neoplasm throughout the world, predominantly reported in growing countries like India, Sri Lanka, Pakistan, Philippines, Vietnam and Brazil which comprises to about $25 \%$ of various cancer types [1]. In the last two decades, next to standard treatments like surgery and radiation, chemotherapy is considered as a systemic treatment for cancers as the chemotherapeutic drugs travel throughout the body and destroys malignant cancer cells spread all over the body away from primary affected site [2]. The chemo drugs used in chemotherapy exhibits its potential in destroying the malignant tumor cells as well as the proliferating somatic cells also, causing to adverse reaction effects [3].

Herbal medicines have been globally accepted over the past decade, and they influence both global health and international trade. Thus, medicinal plants continue to play an important role for a large number of the world's population in the health care system [4]. In recent years, the exploration and usage of drugs and dietary supplements derived from medicinal plants has increased. Scientists such as botanists, microbiologists, Biotechnologist, pharmacologists and phytochemists are involved in utilizing the naturally available phytochemicals that could be transformed into medicines for the treatment of various diseases [5]. There are several medicinal plants used by the physicians of the Indian system of medicine to treat various kinds of diseases [6]. Among the different kinds of medical plants, Polygonum cuspidatum has been practiced for a long period in history in Indian, Chinese, and Japanese traditional and alternative medicine. The pharmacological activity of this plant is due to the presence the presence of biologically active compounds such as Polydatin, emodin, anthraglycoside, and Resveratrol, etc [7]. Polydatin (Resveratrol 3-beta-mono D-glucoside) is a naturally occurring phenolic glycosidic phytocompound abundantly present in P.cuspidatum. Since it was a glycoside type of resveratrol, the pharmacological impact on the cardiovascular system is more important than that of resveratrol [8]. Available literature revealed that that polydatin has diverse of pharmacological activities including

Copyright: $\odot$ The authors. This article is open access and licensed under the terms of the Creative Commons Attribution License (http://creativecommons.org/licenses/by/4.0/) which permits unrestricted, use, distribution and reproduction in any medium, or format for any purpose, even commercially provided the work is properly cited. Attribution - You must give appropriate credit, provide a link to the license, and indicate if changes were made. 
hepatoprotective effects, effects on cardiac muscle cells, lungprotective effects, neuroprotective activity, antitumor activity, anti-inflammatory activity, antimicrobial activity and inhibition of melanin production [9].

Though numerous phytocompounds derived from medicinal plant extracts exists, utilization of the natural compound in therapeutics is limited. This might be a reason to promote a unique practice in distribution of drug, which targets a structured drug delivery pattern that enhances the drug bioavailability in cancer treatment and regulation [10]. Currently in the medical advancement, a novel technology involving effective distribution of nanoparticles that enhances the complete availability of bioactive compounds to exert their hidden chemo preventive property has gained attention [11]. The major advantage is that the nanoparticles are able to conjugate or encapsulate the therapeutic agents and provide betterments over free drugs, with increased half-life, reduced drug toxicity and enhanced targeting efficiency acting as an ideal antineoplastic drug with no side effects [12]. Based on the above studies related to cancer preventive potential of polydatin, our study designed to study the cancer preventive effect of Polydatin encapsulated PLGA nanoparticles against DMBA administered oral carcinogenesis in experimental animals.

\section{MATERIALS AND METHODS}

\section{Chemicals}

DMBA, were procured from Sigma chemical Co., (St. Louis, USA). Other essential reagents and chemicals of analytical grade were supplied from Himedia laboratories, Bangalore, India.

\section{Animals and Bio Formulation of Polydatin Loaded Nanoparticles}

Male golden Syrian hamsters aged about 50 - 60days were bought from National Institute of Nutrition, Hyderabad and they were housed in plastic cages. Experimental protocol was approved by the Institutional Animal Ethical bearing register No. (DKM/IAEC/III/10/2019) and utmost care and supervision was taken in handling animals during entire experimental period. The animals were maintained under restricted environment conditions provided $12 \mathrm{hr}$ dark/light cycle. Polydatin enveloped PLGA nanoparticles (POL-PLGA-NPs) were prepared by oil: water emulsion method and its physiochemical features were confirmed by Fourier transform infrared spectroscopic analysis, dynamic light scattering, atomic force microscope and transmission electron microscope [13].

\section{Experimental Animals}

The animals were alienated to four different experimental sets of six Hamsters in each group. Control animals were labelled as Group I. Animals from II and III was treated with DMBA $(0.5 \%)$ mixed with liquid paraffin weekly three times on left side of Hamster's buccal pouches for a period of 14 weeks to initiate carcinoma in the oral cavity. No other treatment was given to group II animals. Group III Hamsters were orally treated with POL-PLGA-NPs $(30 \mathrm{mg} / \mathrm{kg}$ body weight; dissolved in $0.5 \%$ DMSO) just a week before the carcinogen exposure, continued with DMBA exposure on alternate days till euthanasia was performed. Conversely, group IV Hamsters were administered only with POL-PLGA-NPs to prohibit lethal cases. On completion of stipulated weeks, procedure of cervical decapitation was followed to sacrifice the animals. Tissue samples from buccal cavity of both control and tumor tissues was dissected and proceeded for biochemical and histological analysis.

\section{Tumor Study}

Tumor study was carried out by following Geren et al., (1972) method [14]. Initially, incidence, size and location of tumor were recorded. Following observation of tumor formation, tumor volume and tumor burden were accounted and each tumors diameter were measured by a Vernier caliper. A developed solid cancerous mass that was regarded as prelate ellipsoid with one long axis and two short axes was also measured with Vernier caliper.

The following formula was used to calculate the tumor volume, $\mathrm{V}=\frac{4}{3} \pi\left(\frac{\mathrm{Dl}}{2}\right)\left(\frac{\mathrm{D} 2}{2}\right)\left(\frac{\mathrm{D} 3}{2}\right)$ Tumor diameters are represented as $\mathrm{D}_{1}, \mathrm{D}_{2}$, and $\mathrm{D}_{3}$ (in $\mathrm{cm}$ ).

\section{Biochemical Determination}

Biochemical estimations were performed based on the measurement of absorbance in blood, liver and buccal tissue samples of both control and experimental animals in each group. The Lipid peroxidation activity was detected by the occurrence of thiobarbituric acid reactive substances (TBARS) using a method of Yagi (1987) and the activity was expressed as nmoles/ml [15]. Adopting Jiang et al., (1992) method were used to analysis the Lipid hydroperoxides levels and the result value is recorded in $\mathrm{mmol} / \mathrm{dL}$ of plasma or $\mathrm{mmol} / \mathrm{l} 00 \mathrm{~g}$ of tissues [16]. Conjugated dienes concentration was observed in $233 \mathrm{~nm}$ and result is recorded in $\mathrm{mmol} / \mathrm{dL}$ plasma or mmol/100 $\mathrm{mg}$ tissue or $\mathrm{nmol} / \mathrm{mg}$ of protein for erythrocytes [17]. The method of Kakkar (1984) was used determining the superoxide dismutase activity in plasma and buccal tissue [18]. The method of Rotruck et al., (1975) was utilized to determining glutathione peroxidase activity, the reaction depending on glutathione reaction with, 5'-dithiobis-(2-nitrobenzoic acid) observed at $412 \mathrm{~nm}$ [19] whereas the reduced glutathione levels were determined by following Beuter and kelly's method (1963) [20]. Sinha (1972), developed a method of determining the catalase activity based on the liberation of $\mathrm{H}_{2} \mathrm{O}_{2}$ per minute/ $\mathrm{mg}$ protein in plasma and buccal tissue and the results are recorded in $\mathrm{mg} / \mathrm{dl}$ for plasma and $\mathrm{mg} / 100 \mathrm{mg}$ tissue for buccal cavity [21]. Omura and Sato (1964) framed the method for measuring the drug metabolizing activities of Cytochrome P450 and cytochrome b5 and expressed values in $\mu \mathrm{mol} / \mathrm{mg}$ protein [22]. To determine the activity of Glutathione reductase (GR), Carlberg and Mannervik (1975) method was used [23]. Habig et al., (1974) and Fiala et al., (1972) method was utilized 
to determine the levels of Glutathione S-transferase (GST) activity and G-glutamyl transpeptidase (GGT) activity in which g-glutamyl-p-nitroanilide acts as a substrate, results were indicated as micromoles / gram of tissue per hour [24,25]. The activity of Vitamin $\mathrm{C}$ and vitamin $\mathrm{C}$ were estimated by the method described by Omaye et al., (1979) and Palan et al., (1991) method. The values are noted and expressed in $\mathrm{mg} / \mathrm{dl}$ for plasma and $\mathrm{mg} / 100 \mathrm{mg}$ tissue for buccal cavity $[26,27]$.

\section{Statistical Analysis}

The experimental data was statistically carried out by adopting on ANOVA (one-way analysis of variance). Duncan's Multiple Range Test (DMRT) were employed to distinguish the differences between treatment and control groups using IBM SPSS 20 (SPSS, Tokyo, Japan).

\section{RESULTS}

The tumor incidence, volume, burden and pathological modifications in control and experimental groups were presented in Table 1 and figure 1. Noticeably, 100\% tumor incidence was found in DMBA painted animals. A well-defined squamous cell carcinoma that appeared to be exophytic, large in size with tumor burden of $401.01 \mathrm{~mm}^{3}$ was identified on histological examination. On introduction of POL-PLGA-NPs to DMBA induced HBP, there was a considerable decrease in the mean tumor burden to $76.13 \mathrm{~mm}^{3}$ which indicates a drastic reduction in the tumor burden substantiating the effectiveness of POL-PLGA-NPs towards the metastasis of cancer cells. On histopathological examination, varying degrees of preneoplastic and neoplastic lesions were observed which indicates the presence of mild to moderate hyperplasia.

Table 2 expresses the elevated levels of TBARS, LOOH and CD levels of plasma and erythrocyte, diminished level of the specified parameters in buccal tissue of DMBA induced hamsters as compared to that of the control. On comparing TBARS, LOOH and CD levels of POL-PLGA-NPs (30 mg/kg. b wt.) administered to DMBA exposed animals to that of the control, the results were notably close to the normal levels. This corroborates our previous study, where we studied on treatment of POL-PLGA-NPs was more prominent, as significant activity noticed in DMBA exposed hamsters in a dose-dependent manner. The animals treated with POL-PLGA-NPs alone exhibited no remarkable changes as of the control animals.

Similarly, in Table 3, the parameters of SOD, CAT and GPx are expressed in as reduced levels in Plasma, erythrocyte and buccal

Table 1: Incidence of oral neoplasm and histological changes in the control and experimental animals in each group

\begin{tabular}{|c|c|c|c|c|}
\hline Parameters & Control & DMBA & DMBA+ POL-PLGANPs (30 mg/kg.b.wt) & POL-PLGANPs alone (30 mg/kg.b.wt) \\
\hline Tumor incidence & - & $100 \%$ & $47 \%$ & - \\
\hline Total number of tumors /animals & - & $11 /(6)$ & $3 /(5)$ & - \\
\hline Tumor volume (mm3) & - & $401.01 \pm 39.13$ & $76.13 \pm 8.02$ & - \\
\hline Tumor burden & & $1265.78 \pm 10.99$ & $103.45 \pm 9.16$ & \\
\hline Keratosis & No change & Severe & Mild & No Change \\
\hline Hyperplasia & No change & Severe & Mild & No Change \\
\hline Dysplasia & No change & Severe & Mild & No Change \\
\hline Squamous cell carcinoma & No change & Well differentiate & Moderately differentiated & No Change \\
\hline
\end{tabular}

Data are expressed as the mean \pm SD for 6 hamsters in each group. Values not sharing a common superscript letter in the same row differ significantly at $p<0.05$ (DMRT). Tumor burden was calculated by multiplying tumor volume and the number of tumor/animals. () indicates total number of animals bearing tumors

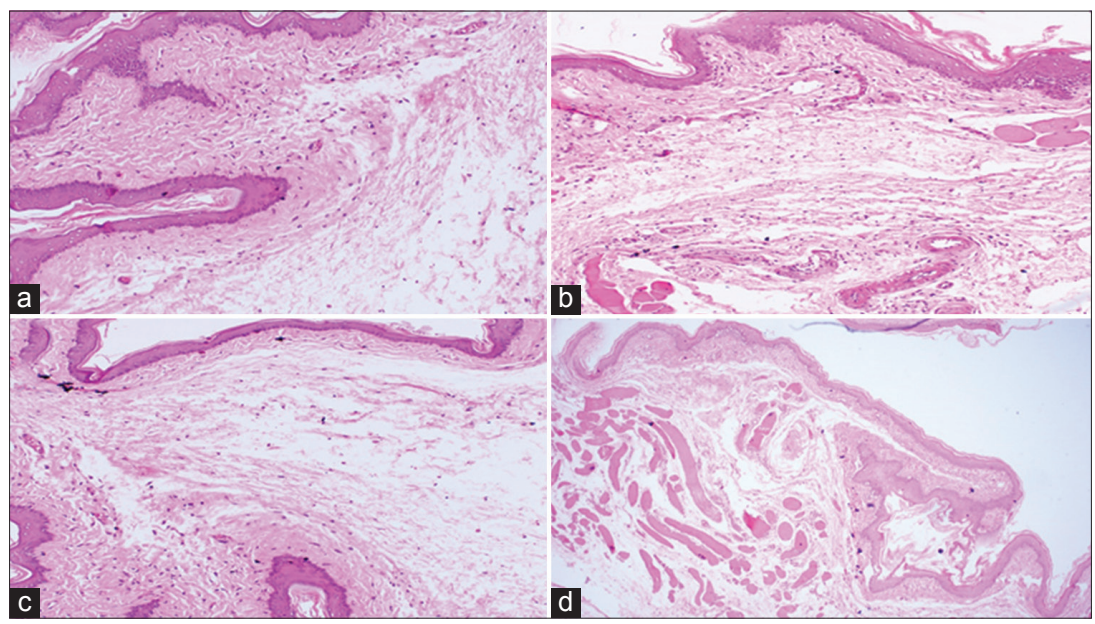

Figure 1: Photomicrographs of histopathological changes were observed in the buccal pouch mucosa of control and experimental hamster in each group. (a\&d) Photomicrograph showing normal epithelium from control and POL-PLGANPs alone treated hamsters (Group 1 and IV). Photomicrograph showing well differentiated squamous cell carcinoma in DMBA alone treated hamsters. (Group II). Photomicrograph showing mild hyperplasia and dysplasia in DMBA+ POL-PLGANPs treated hamsters (Group III). 
mucosa of DMBA exposed hamsters but notably the GPx level in buccal tissue of POL-PLGA-NPs (30 mg/kg. wt) treated DMBA exposed animals is remarkably increased. This indicates that administration of POL-PLGA-NPs (30 mg/kg. wt.) was detected to exhibit more potential as to that of the control. There were no remarkable changes identified with POL-PLGA-NPs alone treated animals to that of the set I(control) animals.

Table 4 demonstrates elevated levels of GSH, Vit-C and Vit-E in buccal tissue of DMBA exposed POL-PLGA-NPs (30 mg/kg b. wt.) treated animals and decreased levels of same specified parameter in plasma and erythrocyte studies when compared to that of the control. On treatment of POL-PLGANPs (30 mg/kg. wt.) to DMBA induced Hamsters, it was observed to produce significant activity in particularly specified group and found more effective as that of the control animals. On POLPLGA-NPs alone treated animals, remarkably no noticeable changes were identified as compared to that of the control.

The modified levels of phase I and Phase II detoxification enzymes are illustrated in Table 5. Phase I enzymatic levels of Cyt $\mathrm{P}_{450}$ and Cyt $\mathrm{b}_{5}$ are observed in an elevated level in buccal mucosa and liver tissue of POL-PLGA-NPs (30 mg/kg b. wt.) treated DMBA exposed Hamsters when compared to that of the control. Whereas an increase in the phase II detoxifying enzymes such as GST, GGT and GR were noted in POL-PLGANPs (30 mg/kg b. wt.) treated DMBA exposed Hamsters' buccal tissue, however, in the liver tissue the level of same specified parameter is noted to be reduced as compared to that of the control. On intragastric administration of $30 \mathrm{mg} / \mathrm{kg}$ b. wt. of POL-PLGA-NPs on DMBA painted Hamsters, levels of phase I and phase II detoxification enzymes were observed near the normal levels as compared to that of the control. Group IV animals treated with POL-PLGA-NPs alone produced no significant changes to that of the control animals.

\section{DISCUSSION}

Oral cancers are a cancerous condition existing in groups collectively described as head and neck cancers. It causes 1:5 mortality in newly diagnosed individuals; 5 years survival rate is slightly more than half [28]. Chemoprevention is the hopeful way for hindering the existence of premalignant lesions to infiltrating cancer. Number of experimental studies are wellgrounded that polydatin can be utilized as a chemo-preventive compound to reverse oral carcinogenesis mechanism [13]. Despite of the broad spectrum of pharmacological properties, use of polydatin in clinical fields is limited due to its property of low aqueous solubility and slow dissolution rate from solid forms. Hence, the usage of polydatin in cancer treatment is limited. In recent years, use of polymer-based nanoparticles in cancer treatment is gaining importance and this platform may enhance the therapeutic values of antitumor drugs by increasing their solubility in aqueous condition such as biologically available and expressing permeability in the tumor environment [29]. Clinical studies demonstrated that nano encapsulated phytochemicals are playing role in reducing the toxicity of chemotherapeutic drugs and facilitate the target

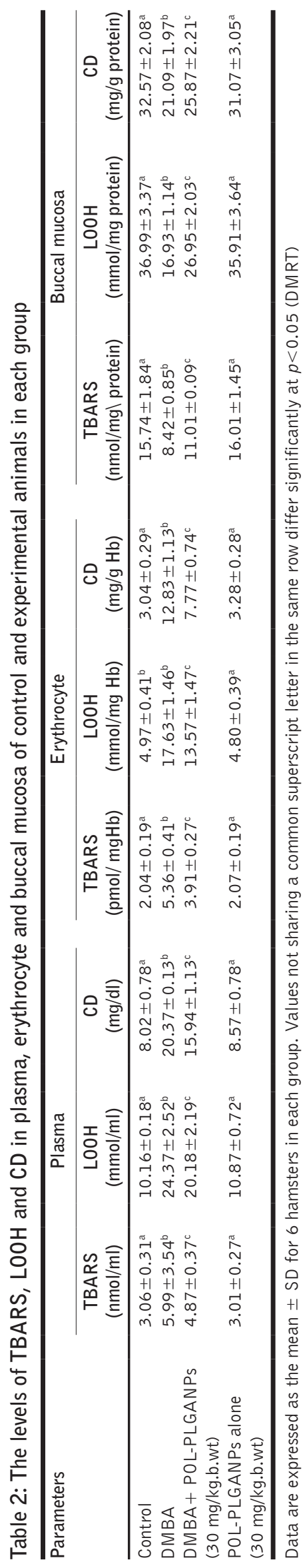

J Phytol • 2020 - Vol 12 
Sankaran, et al.
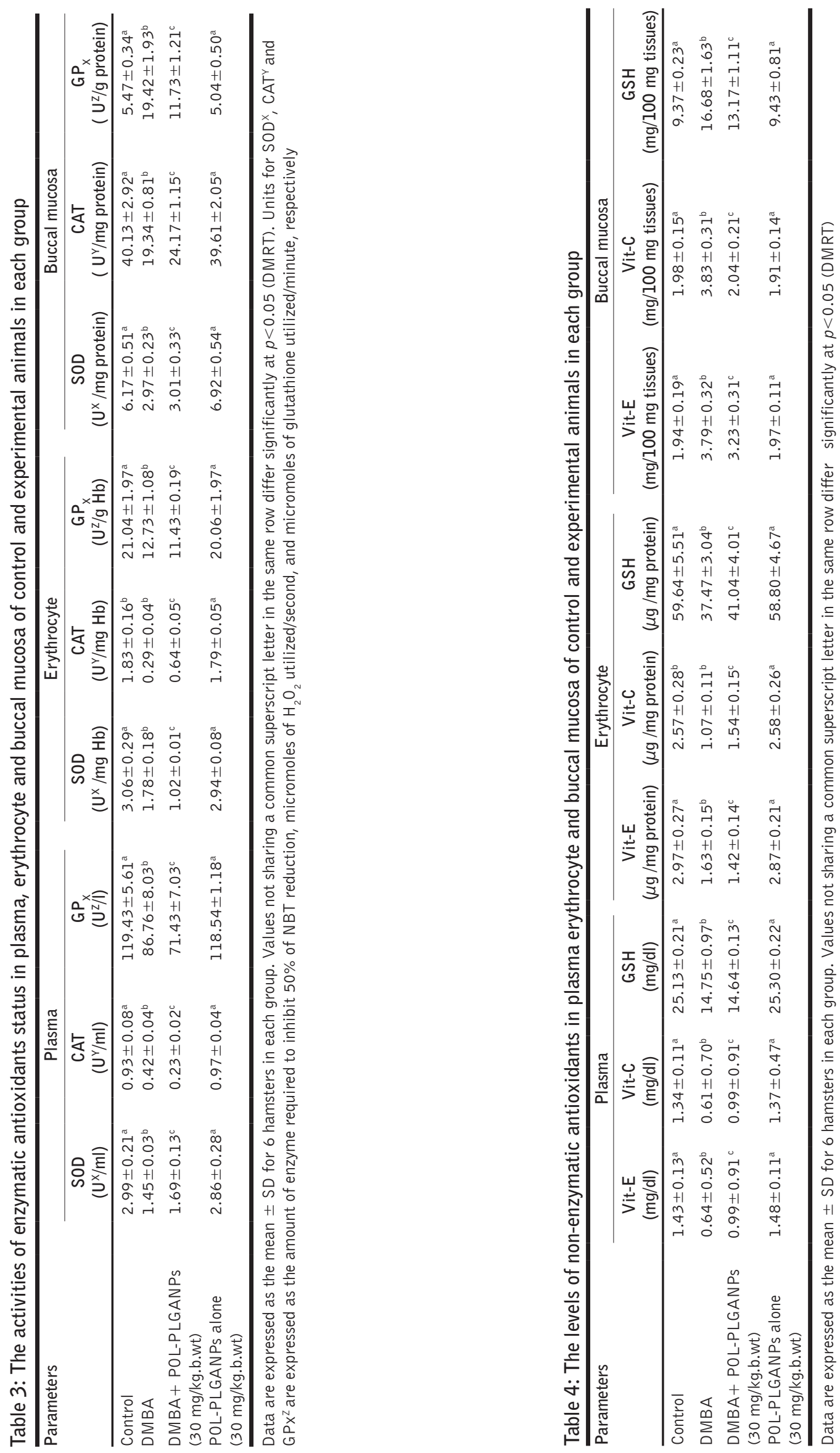


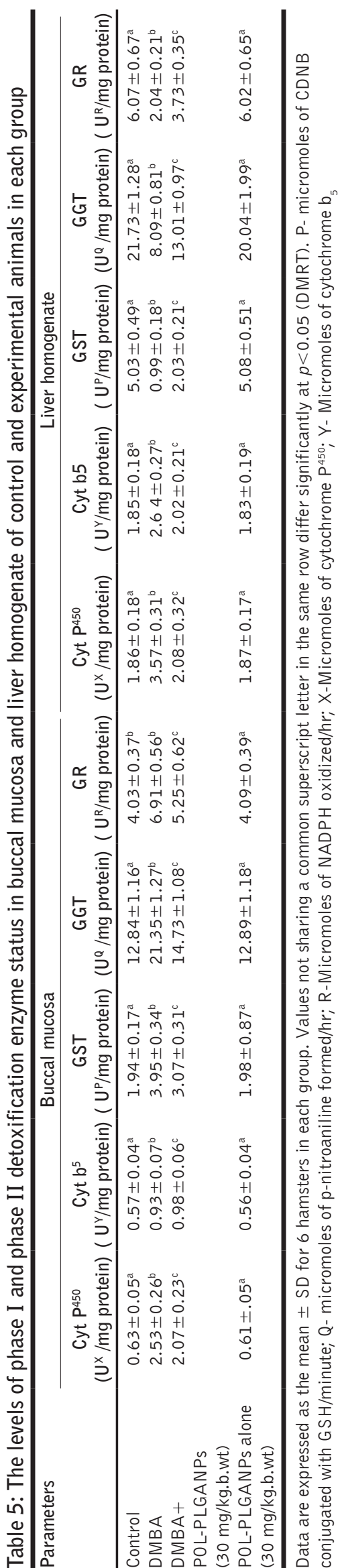

drug delivery [30]. Based on this research findings, we explored the chemo preventive role of PLGA encapsulated polydatin, (a polyphenolic phytocompound present in grape, peanut, hopes cones and cocoa) against DMBA interceded inflammative, cellular proliferation and angiogenesis in hamsters buccal pouch carcinogenesis.

The development of tumors in Hamster buccal pouch exhibits various similarities such as shape, size, pathological features, pre-tumor lesions, property to spread and destruct,, biochemical and molecular markers expression and alterations in genomes as expressed in human OSCC [31]. In Hamster buccal pouch carcinogenesis, a sequential progression is observed through various degrees of dysplasia that is from hyperplasia to invasive carcinoma [32]. In DMBA induced hamsters 100\% formation of tumor occurrence, tumor mass, tumor size and tumor progress were noted and subsequently counterchecked by performing histopathological analysis in which conditions like hyperplasia, dysplasia and squamous cell carcinoma are revealed $[33,34]$. Under favorable conditions, free radicals are expected to be formed within the cell compartments which in turn may be destructed by antioxidant systems. The potent known carcinogen DMBA, interrupts with the pro-oxidant and antioxidant balance leading to depletion of antioxidants of cells/tissues. On the other hand, intragastric tubulation of POL-PLGA-NPs (30 mg/kg b.wt.) on DMBA exposed hamster exhibited significant depleted levels of tumor incidence and tumor volume.

The oxidizing phase I metabolizing enzymes (Cytochrome p450) has a major role in oxidative metabolic potential. Though the metabolic activation of DMBA triggers ROS and RNS, which tend to damage lipids, protein and nucleic acids on application of POL-PLGA-NPs ( $30 \mathrm{mg} / \mathrm{kg}$ b. wt.) to DMBA painted animals, the phase I and phase II enzymes are brought near the normal range as compared to that of the control in buccal mucosa and liver tissues. Also, POL-PLGA-NPs decreases the glutathione utilization and further obstructs the progression of tumorogenesis. Studies on polydatin also reports the effects on glucose and lipid metabolism via protein kinase B (Akt) signaling pathway. AMP-activated protein kinase (AMPK) can be well defined as a significant intracellular sensor which controls glucose and lipid homeostasis. Polydatin counteracts with protein kinase B increasing the AKT phosphorylation thus protecting from enzymatic oxidation [35]. In addition, earlier studies demonstrated that polydatin causes oxidation stress by intervening with receptive oxygen species (ROS) and mitochondrial membrane function. This property of polydatin in the lipid control has gained considerable importance in lipid peroxidation. Thus, it is well-defined that on administration of polydatin, it initiates the AMPK pathway and triggers lipid digestion leading to apoptosis when carried out in trial oral cancer models.

The present study revealed the elevated levels of TBARS, LOOH and CD levels in plasma and erythrocyte and diminished level on specified parameters in buccal tissue of DMBA induced Hamsters. The reason behind is the excessive production of ROS and weakened immune 
response in carcinoma evolved Hamsters. On oral intragastric administration of POL-PLGA-NPs (30 mg/kg.b. wt.), would arrest the disruption of lipid molecules thereby provoking the immunological response in the DMBA painted animals. Based on a survey, the report states that around $60 \%$ of approved antineoplastic drugs are the medicinal plant derivates. The naturally occurring phytochemical enhances GST, GR and GSH (phase II detoxification enzymes) thus exhibiting an increase in their level and diminished phase I enzymatic levels of Cyt P450 and Cyt b5 (29,30). The same or nearer range was observed in our study on oral intragastric application of POL-PLGA-NPs $(30 \mathrm{mg} / \mathrm{kg}$.b. wt.) against the DMBA exposed animals. The predominant non-enzymatic antioxidants such as vitamins $\mathrm{C}$ and $\mathrm{E}$ are abundantly present in phenolic acids and flavonoids of plant derivatives that are rich in antioxidants. This antioxidant activity is decided by electron delocalization over an aromatic nucleus based on a phenolic structure. The structured compounds react with a free radical thereby scavenging the phenolic antioxidant and halts the continuation of the free radical chain reaction. It is regarded as one among the significant antioxidant properties exhibited by polyphenolic compounds [36].

The Phase II enzymatic antioxidants such as CAT, SOD and glutathione peroxidase (GSH-Px) are involved in playing a vital role in guarding cell defence by upgrading the removal of ROS thus providing a defending role against oxidative stress [37]. Further exposure to polydatin increases the expression of Nrf2 gene (Nrf2 is a transcription factor that activates phase II enzymes) through the cis-antioxidant response element (ARE) such that the altered levels of such as SOD, CAT and GSH-Px can be studied in both in vitro and in vivo studies on animals (Hamster). Further, CAT or GPx converts $\mathrm{H}_{2} \mathrm{O}_{2}$ into water, that causes no harm. In cancerous condition both CAT and GPx are known to be depleted than the normal and in the presence of $\mathrm{H}_{2} \mathrm{O}_{2}$, the oxidation of GSH to GSSG is catalyzed by GPx. The result reveals show the decreased level of CAT in cancer bearing animals might be due to the use of antioxidant enzymes in the removal of $\mathrm{H}_{2} \mathrm{O}_{2}$ by DMBA, a potent chemical carcinogen.

Earlier studies on TBARS activity, enzymatic (SOD, CAT and GPx) and non-enzymatic (vitamin E, vitamin C and GSH) antioxidants in DMBA group of buccal pouch carcinogenesis, the result evidenced the increase of level of TBARS and enzymatic antioxidants and depleted in the level of non-enzymatic antioxidants in the plasma of cancer bearing animals [38]. The effects were found to be dose dependent type. In the similar manner, our study revealed that Phase I enzymatic levels of Cyt $\mathrm{P}_{450}$ and $\mathrm{Cyt}_{5}$ are expressed in an elevated level in buccal mucosa and liver tissue of POL-PLGA-NPs (30 mg/kg b.wt) treated DMBA exposed animals whereas an increase in the phase II detoxifying enzymes such as GST, GGT and GR were noted in POL-PLGA-NPs $(30 \mathrm{mg} / \mathrm{kg}$ b.wt $)$ treated DMBA exposed animals' buccal tissue, however, in the liver tissue the level of same specified parameter is noted to be reduced as compared to that of the control. On intragastric administration of $30 \mathrm{mg} / \mathrm{kg}$ b. wt. of POL-PLGA-NPs on DMBA painted Hamsters, detected levels of phase I and phase II detoxification enzymes were observed close to the normal levels as compared to that of the control. This may be due to the resultant of chemo preventive and antioxidants potential of Polydatin.

\section{CONCLUSION}

Animal studies was carried out to rule out the dose dependent study of biofabricated POL-PLGA-NPs and to corroborate the findings of confined dose of POL-PLGA-NPs $(30 \mathrm{mg} / \mathrm{kg}$ b. wt) against DMBA induced oral carcinogenesis with that of the POL-PLGA-NPs (treated alone) and to that of the control levels, which induces oxidative stress mediated tumorogenesis. Based on the findings, it is substantiated that the POL-PLGA-NPs (30 mg/kg b. wt) exhibits the antioxidant and detoxification mechanisms thereby blocking tumor incidence and decreasing tumor volume in DMBA induced experimental animals.

\section{REFERENCES}

1. Tiyuri A, Mohammadian-Hafshejani A, Iziy E, Gandomani HS, Salehiniya $\mathrm{H}$. The incidence and mortality of lip and oral cavity cancer and its relationship to the 2012 Human Development Index of Asia. Biomedical Research and Therapy 2017;4:1147-1165.

2. Ramalingam S, Belani C. Systemic chemotherapy for advanced nonsmall cell lung cancer: Recent. The oncologist. 2008;13:5-13

3. Rejhová A, Opattová A, Čumová A, Slíva D, Vodička P. Natural compounds and combination therapy in colorectal cancer treatment. European Journal of Medicinal Chemistry. 2018;144:582-94.

4. Karunamoorthi K, Jegajeevanram K, Vijayalakshmi J, Mengistie E. Traditional medicinal plants: a source of phytotherapeutic modality in resource-constrained health care settings. Journal of EvidenceBased Complementary \& Alternative Medicine. 2013;18(1):67-74.

5. Elham A, Arken M, Kalimanjan G, Arkin A, Iminjan M. A Review of the Phytochemical, Pharmacological, Pharmacokinetic, and Toxicological Evaluation of Quercus Infectoria Galls. Journal of Ethnopharmacology. 2020:113592.

6. Modak M, Dixit P, Londhe J, Ghaskadbi S, Devasagayam TP. Recent advances in Indian herbal drug research guest editor: Thomas Paul Asir Devasagayam Indian herbs and herbal drugs used for the treatment of diabetes. Journal of Clinical Biochemistry and Nutrition. 2007;40(3): 163-73.

7. Zhang H, Li C, Kwok ST, Zhang OW, Chan SW. A review of the pharmacological effects of the dried root of Polygonum cuspidatum (Hu Zhang) and its constituents. Evidence-Based Complementary and Alternative Medicine. 2013;2013.

8. Xiao K, Li R, Sun M, Hou X, Lin F. Polydatin Use in Vascular Dementia. InDiet and Nutrition in Dementia and Cognitive Decline 2015 (pp. 1059-1067). Academic Press.

9. Du QH, Peng C, Zhang $\mathrm{H}$. Polydatin: a review of pharmacology and pharmacokinetics. Pharmaceutical Biology. 2013;51(11):1347-54.

10. Sun T, Zhang YS, Pang B, Hyun DC, Yang M, Xia Y. Engineered nanoparticles for drug delivery in cancer therapy. Angewandte Chemie International Edition. 2014;53(46):12320-64.

11. Rudramurthy GR, Swamy MK. Potential applications of engineered nanoparticles in medicine and biology: An update. JBIC Journal of Biological Inorganic Chemistry. 2018;23(8):1185-204.

12. Mahmoud BS, AIAmri AH, McConville C. Polymeric Nanoparticles for the Treatment of Malignant Gliomas. Cancers. 2020;12(1):175.

13. Vijayalakshmi S, Mariadoss AVA, Ramachandran V, Shalini V, Agilan B, Sangeetha CC, Balu P, Kotakadi VS, Karthikkumar V', Ernest D. Polydatin Encapsulated Poly [Lactic-co-glycolic acid] Nanoformulation Counteract the 7,12-Dimethylbenz[a] Anthracene Mediated Experimental Carcinogenesis through the Inhibition of Cell Proliferation. Antioxidants (Basel). 2019;8(9):375

14. Green, M., Henle, G., and Deinhardt, F. Respiration and Glycolysis of Human Cells Grown in Tissue Culture. Virology, 5: 206-219,1958.

15. Yagi K. Lipid peroxides and human diseases. Chem Phys Lipids. 1987; 45: 337-351.

16. Jiang ZY, Woollard AC, Wolff SP. Lipid hydroperoxide measurement by oxidation of $\mathrm{Fe} 2+$ in the presence of xylenol orange. Comparison with 
the TBA assay and an iodometric method. Lipids. 1991;26(10):853-6.

17. Rao KS, Recknagel RO. Early onset of lipid peroxidation in rat liver after carbon tetrachloride administration. Exp Mol Pathol 1968; 9:271-78.

18. Kakkar P, Das B, Visvanathan PN. A modified spectrophotometric assay of superoxide dismutase. Indian J Biophys. 1984; 21: 130-132.

19. Rotruck JT, Pope AL, Ganther HT, Swanson AB, Hafeman DG, Hockstra WG. Selenium: Biochemical role as a component of glutathione peroxidase. Science. 1973; 179: 588-590.

20. Beutler E, Kelley BM. The effect of sodium nitrate on RBC glutathione. Experientia. 1963; 19: 96-7.

21. Sinha KA. Colorimetric assay of catalase. Anal Biochem. 1972; 17: 389-94.

22. Omura T, Sato R. The carbon monoxide-binding pigment of liver microsomes. I. Evidence for its hemoprotein nature. J Biol Chem. 1964;239:2370-8.

23. Carlberg I, Mannervik B. Glutathione reductase. In: Meister A, editor. Methods in enzymology. New York. Academic press. 1985; p 484-90.

24. Habig WM, Pabst MJ, Jakoby WB. Glutathione S-transferase. The first enzymatic step in mercapturic acid formation. J Biol Chem. 1994; 249: 7130-7139.

25. Fiala S, Fiala AE, Dixon B. Gamma-glutamyl transpeptidase in transplantable chemically induced rat hepatomas and "spontaneous" mouse hepatomas. J Natl Cancer Inst. 1972; 48: 1393-1401.

26. Omaye ST, Turnbull TD, Sauberlich HE. Selected method for the determination of vitamin $\mathrm{E}$ in animal cells, tissues and fluids. In: Mc Cormic DB, Wright DL, Eds. Methods Enzymol. 1979; 62: 3-11.

27. Palan PR, Mikhail BS, Basin J, Romney SL. Plasma Levels of antioxidant beta-carotene and tocopherol in uterine cervix dysplasia and cancer. Nutr. Cancer. 1973; 15: 13-20.

28. Dholam KP, Chouksey GC (2016) Squamous cell carcinoma of the oral cavity and oropharynx in patients aged $18-45$ years: A case-control study to evaluate the risk factors with emphasis on stress, diet, oral hygiene, and family history. Indian J Cancer 53:244.

29. Arokia Vijaya Anand Mariadoss, Ramachandran Vinayagam, Vijayalakshmi Senthilkumar, Manickam Paulpandi, Kadarkarai Murugan, Baojun Xu, Gothandam K.M., Venkata Subbaiah Kotakadi, Ernest David. Phloretin loaded chitosan nanoparticles augments the $\mathrm{pH}$-dependent mitochondrial-mediated intrinsic apoptosis in human oral cancer cells. International Journal of Biological Macromolecules 2019; 130: 997-1008.
30. Sivaraj M, Mukherjee A, Mariappan R, Mariadoss AV, Jeyaraj M Polyorganophosphazene stabilized gold nanoparticles for intracellular drug delivery in breast carcinoma cells. Process Biochemistry. 2018;72:152-61.

31. Suresh K, Manoharan S, Vijayaanand MA, Sugunadevi G. Chemopreventive and antioxidant efficacy of (6)-paradol in 7, 12-dimethylbenz (a) anthracene induced hamster buccal pouch carcinogenesis. Pharmacological Reports. 2010;62(6):1178-85.

32. Rajkamal G, Suresh K, Sugunadevi G, Vijayaanand MA, Rajalingam K. Evaluation of chemopreventive effects of Thymoquinone on cell surface glycoconjugates and cytokeratin expression during DMBA induced hamster buccal pouch carcinogenesis. BMB Reports 2010;43(10):664-69.

33. Kathiresan Suresh, Shanmugam Manoharan, Mariadoss Arokia Vijayaanand, Govindasamy Sugunadevi. Chemopreventive and antioxidant efficacy of (6)-paradol in 7,12-dimethylbenz(a)anthracene induced hamster buccal pouch carcinogenesis. Pharmacological Reports 2010;62(6):1178-85.

34. Arokia Vijayaanand Mariadoss, Suresh Kathiresan, Rajasekar Muthusamy, Sivakumar Kathiresan. Protective effects of [6]-paradol on histological lesions and immunohistochemical gene expression in DMBA induced hamster buccal pouch carcinogenesis. Asian Pacific Journal of Cancer Prevention 2013;14(5):3123-3129.

35. Hao J, Huang K, Chen C, Liang Y, Wang Y, Zhang X, Huang H. Polydatin improves glucose and lipid metabolisms in insulin-resistant HepG2 cells through the AMPK pathway. Biological and Pharmaceutical Bulletin. 2018;41(6):891-8

36. Ammar RB, Bhouri W, Sghaier MB, Boubaker J, Skandrani I, Neffati A, Bouhlel I, Kilani S, Mariotte AM, Chekir-Ghedira L, DijouxFranca MG. Antioxidant and free radical-scavenging properties of three flavonoids isolated from the leaves of Rhamnus alaternus L.(Rhamnaceae): A structure-activity relationship study. Food Chemistry. 2009;116(1):258-64.

37. Apel K, Hirt H. Reactive oxygen species: metabolism, oxidative stress, and signal transduction. Annu. Rev. Plant Biol.. 2004;55:373-99.

38. Mariadoss AV, Vinayagam $R, X u B$, Venkatachalam K, Sankaran V Vijayakumar S, Bakthavatsalam SR, Mohamed SA, David E. Phloretin loaded chitosan nanoparticles enhance the antioxidants and apoptotic mechanisms in DMBA induced experimental carcinogenesis. Chemico-biological interactions. 2019;308:11-9. 